\title{
Improved Sparse NMF based Speech Enhancement Method with Deep Neural Network
}

\section{Xia Zou ${ }^{1}$, Xiongwei Zhang ${ }^{1}$, Wenhua Shi, ${ }^{1,2}$, Fupeng Wang ${ }^{3}$, Jingtao Zhang ${ }^{3}$ and Mingyue $\mathrm{Gao}^{3}$}

\author{
${ }^{1}$ Army Engineering University of PLA, Nanjing 210007, China \\ ${ }^{2}$ Flight Training Base, Air Force Aviation University, Fuxin 123000, China \\ ${ }^{3} 91285$ unit of PLA, Dalian, 116000, China
}

\begin{abstract}
Keywords: Speech enhancement; Deep neural network; Sparse non-negative matrix factorization. Abstract. Considering the sparsity characteristic of speech signal in time-frequency domain and the non-linear model ability of deep neural network, an improved sparse non-negative matrix factorization based speech enhancement method is presented in this paper. Deep neural network is employed to learn the sparse encoding coefficients of speech and noise from noisy observation. The estimated clean speech is obtained by applying the wiener filter on the magnitude spectrogram of noisy speech. The experimental results show the superiority of proposed method under stationary and non-stationary conditions.
\end{abstract}

\section{Introduction}

Speech enhancement is to recover the speech contaminated by background noise. It has been widely used in speech communication, automatic speech recognition and wearable hearing aids. In the past few decades, numbers of speech enhancement methods have been proposed. Traditional approaches including spectral subtraction and wiener filter in frequency domain. Statistical properties based approaches like minimum mean squared error (MMSE) estimation and optimally-modified log-spectral amplitude (OM-LSA) which could take human hearing properties into account and reduce speech distortion and residual noise in some extent ${ }^{[1]}$.

Recent years, supervised learning methods have achieved significant development in speech signal processing ${ }^{[2-3]}$. As a famous method which could mine implicit local representation in non negative data, non-negative matrix factorization (NMF) uses no-negative linear combination to separate clean and noise signal from noisy speech. A limitation of separating different source by linear combination of their basis matrix is due to the none-orthogonal basis vector of each source. When different sound sources are overlapping or share the similar basis matrix, i.e. the target sound source may be the represented by basis matrix of other sound sources, NMF based source separation will not be able to correctly separate each sound source. The authors of [4] use discriminative training of base vectors, and authous of [5] solve this problem by using DNN to learn the non-negative coding matrix from the non-negative magnitude spectrogram. Considering the sparsity characteristic of speech signal in time-frequency domain and the non-linear model ability of deep neural network, an improved sparse non-negative matrix factorization based speech enhancement method is presented in this paper.

\section{Proposed Method}

The flow chart of the proposed method is illustrated in Fig. 1. The method is composed of training stage and enhancement stage. In training stage, sparse non-negative factorization (SNMF) is applied on the magnitude spectrogram of noise and speech to get the basis matrix and encoding coefficient matrix for noise and speech respectively. Then the magnitude spectrogram of noisy speech is used as the input of the DNN and the encoding coefficient matrix of noise and speech is used as the target output of the DNN. Then a supervised learning of the non-linear function which maps the magnitude spectrogram of noisy speech to the encoding coefficient matrix of noise and speech is trained in the second step. In enhancement stage, magnitude spectrogram of noisy speech is fed into the well trained 
DNN model in training stage to get the coding coefficient matrix of noise and speech. Wiener filter is constructed by the basis matrix and coding coefficient matrix. The estimated clean speech is obtained by applying the wiener filter on the magnitude spectrogram of noisy speech.

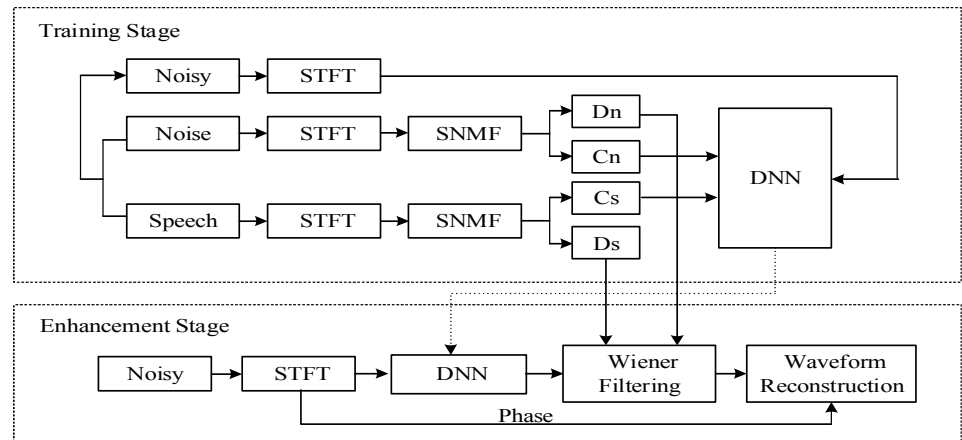

Figure 1 Flow chart of the proposed method

\section{Sparse non-negative factorization (SNMF)}

NMF is using nonnegative dictionary matrix and the product of the coding matrix to achieve the approximation of the overall nonnegative matrix. SNMF is based on the sparsity characteristic of speech signal in time-frequency domain, which makes the model simple, effective, and robust for noise depression ${ }^{[6]}$ :

$$
\mathbf{D}, \mathbf{C}=\arg \min _{\mathbf{D} \geq 0, \mathbf{C} \geq 0} D_{K L}(\mathbf{Y} \mid \mathbf{D C})+\lambda\|\mathbf{C}\|_{1}
$$

Where $\mathbf{Y}$ denotes the magnitude spectrogram of current frame. $\mathbf{D}$ represent the dictionary basis matrix for speech or noise. $\lambda$ is the parameter that controls the sparsity of the coding matrix $\mathbf{C}$, the larger the value of $\lambda$, the more sparse the coding matrix is. $D_{K L}$ is a distance measure describing the approximation extent between the decomposed matrix and the original matrix. The $K L$ divergence has expression as follows:

$$
D_{K L}(\mathbf{A} \mid \mathbf{B})=\sum_{k, n}\left\{\mathbf{A}_{k, n} \log \frac{\mathbf{A}_{k, n}}{\mathbf{B}_{k, n}}-\mathbf{A}_{k, n}+\mathbf{B}_{k, n}\right\}
$$

The problem in (2) could use gradient descent method and solved by multiplicative iteration algorithm:

$$
\begin{aligned}
& \mathbf{C} \leftarrow \mathbf{C} \quad \frac{\overline{\mathbf{D}}^{T} \frac{\mathbf{Y}}{\overline{\mathbf{D}} \mathbf{C}}}{\overline{\mathbf{D}}^{T} \mathbf{1}+\lambda}
\end{aligned}
$$

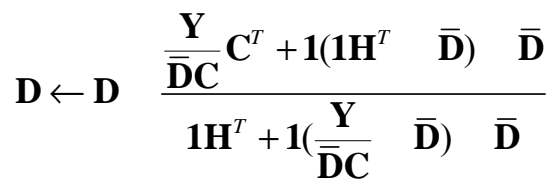

\section{DNN based model training}

A forward neural network with $L$ hidden layers is adopted to learn the non-linear function which maps the magnitude spectrogram of noisy speech to the coding coefficient matrix of noise and speech. The cost function is defined as follows ${ }^{[7]}$ :

$$
J_{M S E}(\mathbf{W}, \mathbf{b})=\frac{1}{N} \sum_{n=1}^{N}\left\|\hat{\mathbf{C}}\left(\mathbf{Y}_{n+K}^{n-K}, \mathbf{W}, \mathbf{b}\right)-\mathbf{C}\right\|_{2}^{2}
$$

Where $\mathbf{W}$ and $\mathbf{b}$ denote the weight matrix and bias matrix of the network respectively, $\mathrm{N}$ is the batch size. $\mathbf{C}=\left[\mathbf{C}_{s} ; \mathbf{C}_{n}\right]$ denotes the target output of the network, $\hat{\mathbf{C}}=\left[\hat{\mathbf{C}}_{s} ; \hat{\mathbf{C}}_{n}\right]$ is estimation of the output. $\mathbf{Y}_{n+K}^{n-K}$ denotes the $2^{*} K+1$ frames magnitude spectrogram centered on the current frame. 


\section{Wiener filter and reconstruction}

After the coding coefficient matrix of noise and speech is obtained from the output of the network, the magnitude spectrogram of the enhanced speech could be calculated by using the wiener filtering or time-frequency masking method ${ }^{[10]}$ :

$$
\hat{\mathbf{Y}}=\frac{\mathbf{D}_{s} \mathbf{C}_{s}}{\mathbf{D}_{s} \mathbf{C}_{s}+\mathbf{D}_{n} \mathbf{C}_{n}} \quad \mathbf{Y}
$$

Since speech is not sensitive to the phase information, by appending the phase information of the noisy speech signal to the estimated magnitude spectrogram, the time domain speech signal could be reconstructed using inverse short time Fourier transform (STFT).

\section{Experimental and results}

In this experiment, 7200 noisy speech are generated by adding six different types of noise (i.e. babble, f16, factory, pink, white and hf channel) from Noise- $92^{[8]}$ database to random selected clean sentences form TIMIT ${ }^{[9]}$ training subset. $10 \%$ of the training set is selected as the verification or development set. The best network parameters are selected by the results on the verification set. Another 480 utterances from TIMIT test subset are mix with the six types of noise to evaluate the performance of the proposed method in terms of perceptual evaluation of speech quality (PESQ $)^{[10]}$ and log-spectral distance (LSD) $)^{[11]}$.

All the speech and noise signals are down-sampled to $8000 \mathrm{~Hz}$. The magnitude spectrogram of clean speech, noise signal and noisy speech are extracted using a 256 point STFT with a frame shift of 64 point. In NMF based speech enhancement method, the speech and noise dictionary size of $\mathbf{D}_{\mathrm{s}}$ and $\mathbf{D}_{\mathrm{n}}$ is set to 256. In SNMF, the sparsity factor $\lambda$ is set to 0.01 .500 multiplicative iterations are performed to get the basis matrix and coding coefficient matrix. In DNN, a feed forward network with 3 hidden layers of 1024 neurons is used to map the noisy magnitude spectrogram to the magnitude spectrogram of clean speech. In the proposed method, a feed forward network with 3 hidden layers of 1024 neurons is employed to map the noisy magnitude spectrogram to the coding coefficient matrix of clean speech and noise. We select the linear activation function at the output layer of the network and select rectified linear function as the activation function of the hidden layers. Compared with the sigmoid function, the rectified linear function is more accordant with the principle of neuron excitation and with sparse output ${ }^{[7]}$.

TABLE I. EVALUATION WITH PESQ/LSD ON NOISY AND ENHANCED SPEECH

\begin{tabular}{|c|c|c|c|c|c|}
\hline \multirow{2}{*}{$\begin{array}{c}\text { SNR } \\
\text { (dB) }\end{array}$} & \multicolumn{5}{|c|}{ PESQ } \\
\hline-5 & 1.213 & 1.392 & 1.356 & 1.456 & 1.528 \\
\hline 0 & 1.519 & 1.745 & 1.712 & 1.890 & 1.836 \\
\hline 5 & 1.864 & 2.107 & 2.078 & 2.224 & 2.138 \\
\hline 10 & 2.216 & 2.467 & 2.439 & 2.454 & 2.449 \\
\hline Ave & 1.703 & 1.928 & 1.896 & 2.006 & 1.988 \\
\hline
\end{tabular}

(b)

\begin{tabular}{|c|c|c|c|c|c|}
\hline \multirow{2}{*}{$\begin{array}{c}\text { SNR } \\
(\mathbf{d B})\end{array}$} & \multicolumn{5}{|c|}{ LSD } \\
\hline-5 & 2.727 & 2.566 & 2.470 & 1.969 & 2.421 \\
\hline 0 & 2.556 & 2.280 & 2.213 & 1.732 & 2.165 \\
\hline 5 & 2.281 & 1.934 & 1.896 & 1.625 & 1.885 \\
\hline 10 & 1.943 & 1.604 & 1.592 & 1.381 & 1.606 \\
\hline Ave & 2.377 & 2.096 & 2.043 & 1.654 & 2.020 \\
\hline
\end{tabular}

The average PESQ and LSD scores of NMF, SNMF, DNN and proposed speech enhancement method at four SNR levels averaged on six types of noise are shown in Table 1. Table 1 gives a comparison of three speech enhancement methods with different SNRs. Compared with NMF and SNMF based speech enhancement methods, our proposed approach has gained better perceptual quality and less speech distortion. Although the proposed method has comparable perceptual quality with DNN based method, the speech distortion is larger than DNN based method.

\section{Conclusions}

Considering the sparsity characteristic of speech signal in time-frequency domain and the non-linear model ability of deep neural network, an improved sparse non-negative matrix factorization based 
speech enhancement method is presented in this paper. Deep neural network is employed to learn the sparse coding coefficients of speech and noise from noisy observation. The estimated clean speech is obtained by applying the wiener filter on the magnitude spectrogram of noisy speech. The experimental results show the superiority of proposed method when compared with NMF and SNMF based approaches.

\section{Acknowledgements}

This work is partially supported by NSF of China (Grant No. 61471394, 61402519), NSF of JIANG Su Province (Grant No. BK20140071, BK20140074).

\section{References}

[1] P. C. Loizou: Speech enhancement: theory and practice (CRC press, 2013).

[2] N. Mohammadiha, P. Smaragdis, and A. Leijon: IEEE/ACM Trans. on Audio, Speech, and Language Processing, vol. 21, no. 10 (2013), pp. 2140-2151

[3] Y. Xu, J. Du, L. R. Dai, and C. H. Lee: IEEE/ACM Trans. on Audio, Speech, and Language Processing, vol. 23, no. 1 (2014), pp. 7-19, 2014.

[4] F.Weninger, J. Le Roux, J. R. Hershey, et al. in Proc. Conference of the International Speech Communication Association, 2014, pp. 543-547.

[5] Nie S, Liang S, Li H, et al: in Proc. of the International Conference on Acoustics, Speech and Signal Processing. IEEE, 2016:469-473.

[6] Eggert J, Korner E: in Proc of the 2004 IEEE Int Joint Conf on Neural Networks. Piscataway, NJ: IEEE, 2004: 2529-2533

[7] Y. X.Wang, A. Narayanan, and D. L.Wang: IEEE/ACM Trans. on Audio, Speech, and Language Processing, vol. 22, no. 12 (2014), pp. 1849-1858

[8] V. Zue, S. Seneff, and J. Glass: Speech Commun., vol. 9, no. 4 (1990), pp. 351-356

[9] A. Varga and H. J. M. Steeneken: Speech Commun., vol. 12, no. 3 (1993), pp. 247-251

[10] Rix A, Beerends J, Hollier M P, et al: in Proc of the 26th IEEE Int Conf. on Acoustics, Speech and Signal Processing. Piscataway, NJ: IEEE, 2001:749-752

[11] Du J, Huo Q: in Proc of the 33th IEEE Int Conf on Acoustics, Speech and Signal Processing. Piscataway, NJ: IEEE, 2008:4721-4724 